\title{
Consistent Quantum Counterfactuals
}

\author{
Robert B. Griffiths* \\ Department of Physics \\ Carnegie Mellon University \\ Pittsburgh, PA 15213
}

June 17, 2021

\begin{abstract}
An analysis using classical stochastic processes is used to construct a consistent system of quantum counterfactual reasoning. When applied to a counterfactual version of Hardy's paradox, it shows that the probabilistic character of quantum reasoning together with the "one framework" rule prevents a logical contradiction, and there is no evidence within this approach for any nonlocal influences. Counterfactual reasoning can support a realistic interpretation of standard quantum theory (measurements reveal what is actually there) under appropriate circumstances.
\end{abstract}

A bullet fired at a beer mug is stopped by a wooden board located between the gun and the mug. What would have happened if the board had not been present? The answer to simple counterfactual questions of this sort is intuitively obvious for events in the macroscopic "classical" world of everyday experience. Their quantum counterparts, on the other hand, have given rise to endless controversy. Suppose a Stern-Gerlach apparatus measures $S_{z}=1 / 2$ for a spin half particle. Would $S_{z}$ have been $1 / 2$ if the measurement had not been made? What would have been the result if the apparatus had been set up to measure the spin in a different direction? Readers familiar with the EPR paradox and Bell's inequality [1, 2, 园, 四, Hardy's paradox [5], and the like are probably aware that even admitting that such counterfactual questions might have answers can be a dangerous first step into a conceptual swamp [6]. Nonetheless, counterfactuals seem a necessary part of any realistic version of quantum theory in which properties of microscopic systems are not simply "created" by measurements [7]. And if our everyday experiences take place in a world which is fundamentally quantum mechanical, as most physicists believe, there must be at least some cases of valid quantum counterfactuals having to do with boards and bullets and the like.

This article will show how to construct a limited system of quantum counterfactual reasoning able to address the issues mentioned in the preceding paragraph. It extends smoothly into the "classical" world of everyday experience, where it gives intuitively sensible answers; alternatively, it represents a possible way to extend some types of classical counterfactual reasoning [8] into the quantum realm, without fear of generating contradictions. It provides

${ }^{*}$ Electronic address: rgrif@cmu.edu 

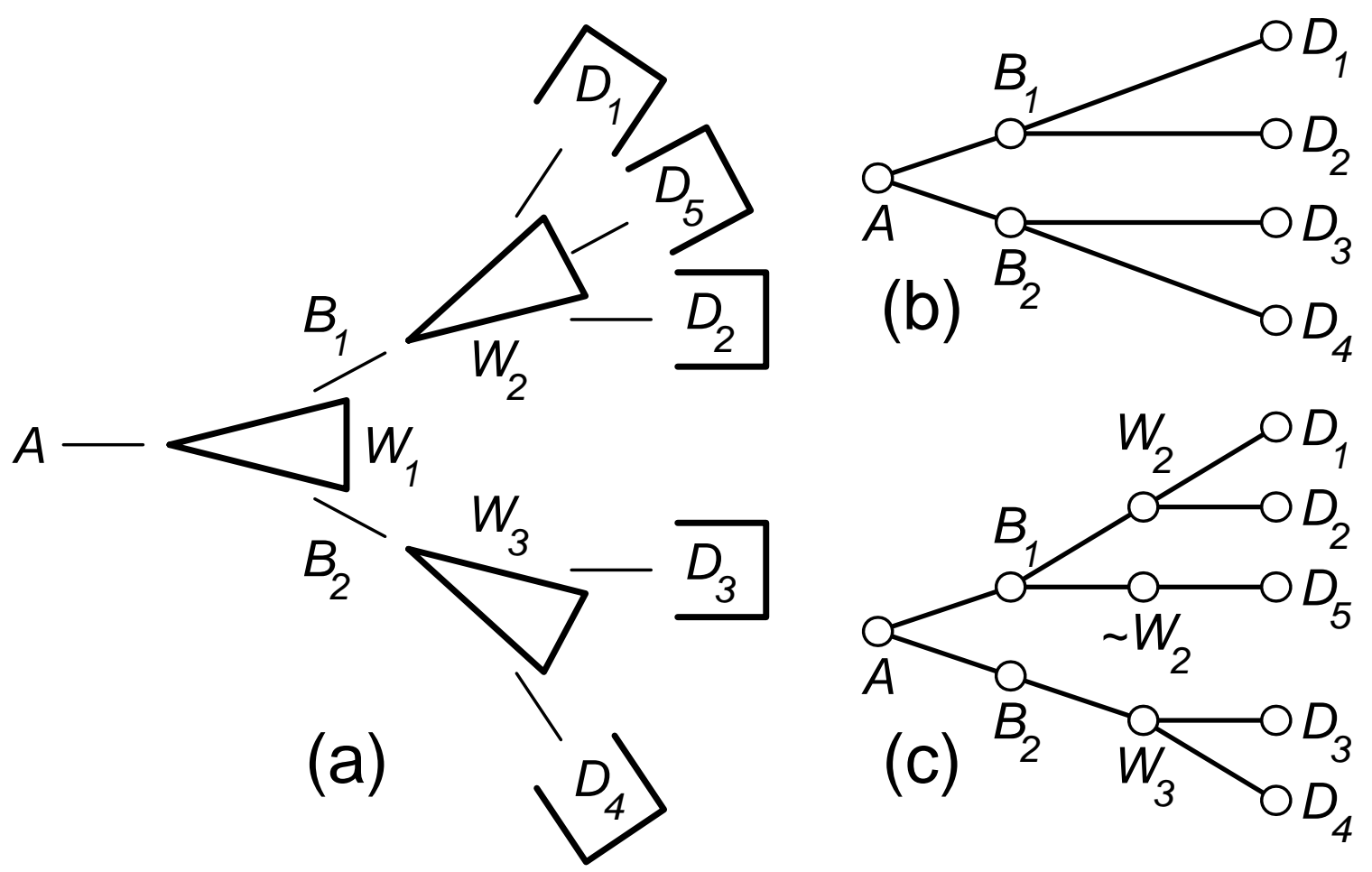

Figure 1: (a) Particle scattering from wedges. (b) Schematic representation of possible histories. (c) Histories with wedge $W_{2}$ present or absent $\left(\sim W_{2}\right)$.

a way of understanding a counterfactual version of Hardy's paradox without coming to the conclusion that quantum theory involves mysterious nonlocal influences. And it can serve as one element of a realistic interpretation of quantum theory. In its present form it is restricted to non-relativistic quantum mechanics.

We begin by considering the classical stochastic process shown in Fig. 1(a): a particle starting at $A$ moves to the right and scatters randomly off a set of wedges on its way to a series of detectors, $D_{1}, D_{2} \ldots$ Suppose the particle arrives at $D_{1}$. What would have happened if it had suffered a different deflection at wedge $W_{2}$ ? The answer can be read off of Fig. 1(b), a symbolic representation of possible particle trajectories or histories, by starting at node $D_{1}$, moving backwards in time to the pivot point $B_{1}$, the event immediately preceding the particle's encounter with $W_{2}$, and then forward in time along the alternative, or counterfactual, branch to $D_{2}$. Similarly, the question "What would have happened had the particle scattered the other way at $W_{1}$ ?" can be answered by using $A$ as the pivot. Going forwards in time from $A$ on the alternative (lower branch) in Fig. 1(b), one sees that two histories, $A B_{2} D_{3}$ and $A B_{2} D_{4}$ are possible, and thus the correct answer to this counterfactual question is not a definite result but instead a probability distribution assigning appropriate weights to these alternatives. Indeed, in a classical stochastic world, the correct answer to a counterfactual question will in general be probabilistic; only in special cases will the probability be one. Even a null counterfactual question, one for which the antecedent actually occurred, can have a probabilistic answer. For example, the answer to: "What would have happened had the particle scattered the same way at $W_{1}$ ?" (that is, in the same direction in which it actually did scatter in order to arrive at $D_{1}$ ) is a probability distribution with both 
$D_{1}$ and $D_{2}$ allowed, rather than $D_{1}$ alone, if we use Fig $1(\mathrm{~b})$ with $A$ as the pivot. That null counterfactuals can have this character is typical of a stochastic, in contrast to a deterministic theory, and has important applications in the case of quantum counterfactuals, as we shall see. The same strategy can be used to address the question "What would have happened if wedge $W_{2}$ had been absent?" given that $D_{1}$ was observed. Assume that a stochastic "coin flip", a purely mechanical process with no human intervention, occurs just before the particle arrives at $W_{2}$. Depending on the outcome, the wedge is left in place or yanked out of the way by a servomechanism. Using Fig. 1(c), with $B_{1}$ as the pivot, one obtains $D_{5}$ as the answer. One can also use $A$ as the pivot, in which case the answer is that either $D_{3}, D_{4}$, or $D_{5}$ would have occurred, with certain probabilities. Both of these results are intuitively plausible responses to the counterfactual question, interpreted in slightly different ways. The fact that they are different shows the importance of identifying the pivot when one uses this approach to counterfactual reasoning.

Our proposal for quantum counterfactual reasoning is to use precisely the same method of analysis applied to a consistent family or framework of quantum histories: sequences of events represented by orthogonal projection operators (projectors) to which probabilities are assigned using the standard dynamical laws of quantum theory [9, 10]. For example, imagine that the particle in Fig. 1(a) is represented by a wave packet which scatters off of successive wedges - or think of a photon passing through a succession of beam splitters. The events $B_{1}$ and $B_{2}$ correspond to projectors on appropriate regions of space. A wedge or other scattering center can be moved out of the way at the last moment based upon a quantum coin flip: think of a photon passing through a beamsplitter before triggering one of two photodetectors connected to suitable amplifiers and a servomechanism. Counterfactual conclusions are then based upon diagrams of the type shown in Fig. 1 in the same way as in the classical case. The main difference between classical and quantum reasoning comes about through the fact that quantum events are described using a Hilbert space, and this allows a multiplicity of stochastic quantum descriptions (frameworks or consistent families or logics) which are mutually incompatible [9, 10]. Following the usual rules for consistent histories, we require that a valid counterfactual argument employ a single framework; in particular, combining results from two incompatible quantum frameworks is not allowed [11].

The multiplicity of possible frameworks gives quantum counterfactual reasoning a slightly different flavor from its classical counterpart, as shown in the following example. Imagine that at time $t_{0}$ a spin half particle with spin in some direction $w$ is traveling towards a Stern-Gerlach apparatus, and at $t_{1}$, shortly before it arrives, a quantum coin is "flipped". Outcome 1 ("heads") results in a servomechanism orienting the apparatus so that at time $t_{2}$ it is in a state $Z$ appropriate for measuring $S_{z}$, while outcome 2 ("tails") results in an apparatus state $X$ for measuring $S_{x}$. At time $t_{3}$ the measurement is complete, and in case 1 (heads) the apparatus is in one of the two states $Z^{+}$or $Z^{-}$, corresponding to a measurement of $S_{z}= \pm 1 / 2$, while in case 2 (tails) the state is $X^{+}$or $X^{-}$, corresponding to $S_{x}= \pm 1 / 2$.

One framework for describing this system is indicated schematically in Fig. 2(a), where the node $A$ at $t_{0}$ represents the initial state of the particle and the apparatus (including the quantum coin), the node $B$ at $t_{1}$ corresponds to the unitary time development of state $A$, and the nodes at $t_{2}$ and $t_{3}$ indicate the apparatus states before and after the measurements. Suppose, for example, that $S_{z}$ is measured with the result $Z^{+}$. What would have happened if the quantum coin flip had yielded 2 , resulting in a measurement of $S_{x}$ ? The answer, obtained 

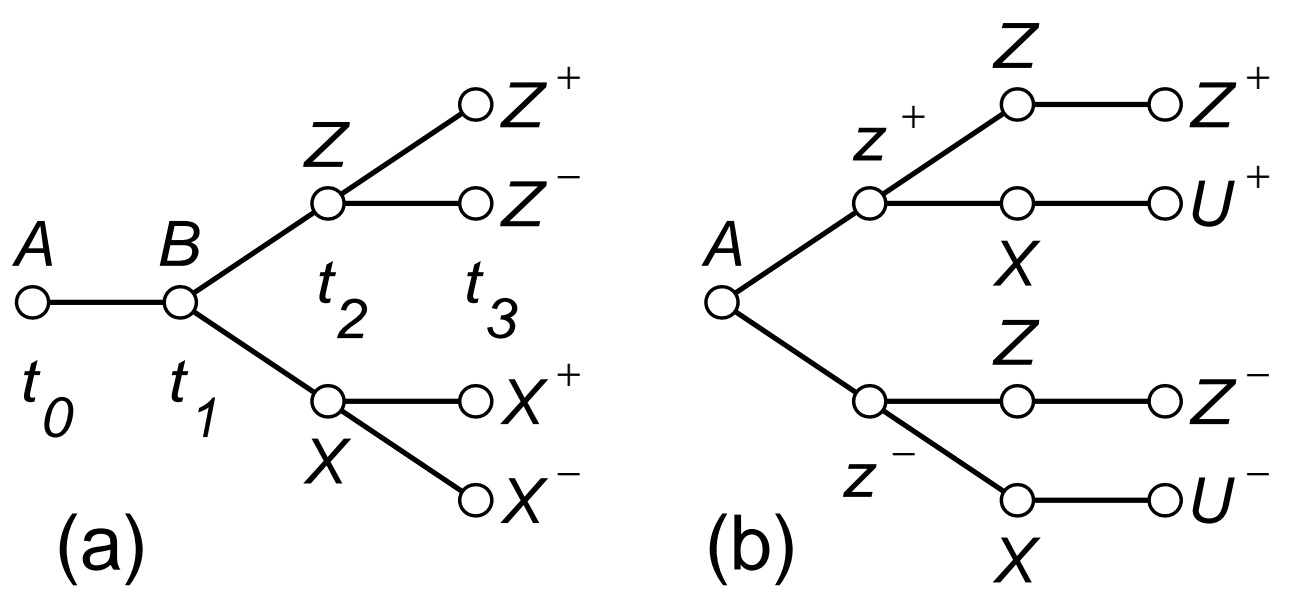

Figure 2: Two consistent families of histories corresponding to measuring spin components of a spin half particle in directions determined by flipping a quantum coin.

by starting at node $Z^{+}$, going backwards to node $B$ (immediately preceding the quantum coin flip), and then forwards on the lower branch is: $X^{+}$or $X^{-}$with a certain probability, depending upon the initial spin direction $w$. This seems intuitively plausible. Applying the same method of reasoning to the null counterfactual question, "What would have happened if the quantum coin flip resulted (as it actually did) in a measurement of $S_{z}$ ?" does not yield the answer $Z^{+}$; instead, there are positive probabilities for both $Z^{+}$and $Z^{-}$(unless the initial spin direction $w$ coincides with $z$ ).

A "sharper" answer to this null counterfactual is provided by the alternative framework in Fig. 2(b) in which the single node $B$ in (a) has been replaced by two nodes $z^{+}$and $z^{-}$ corresponding to particle spin states $S_{z}= \pm 1 / 2$ before the quantum coin is flipped, and thus before the measurement. This framework, which is allowed by consistent histories, though not by ordinary textbook quantum theory, permits one to say that the measurement result $Z^{+}$reflects the prior state, $z^{+}$or $S_{z}=+1 / 2$, of the measured particle [10]. If the node $z^{+}$ is used as the pivot, the answer to the null counterfactual question in a case in which $Z^{+}$ is observed is that $Z^{+}$would have been observed (probability 1) if $S_{z}$ had been measured (as it actually was). However, using this same pivot leads to the conclusion that had the coin resulted in a measurement of $X$ rather than $Z$, the result would have been (again with probability 1) the macroscopic quantum superposition (MQS or Schrödinger cat) state $U^{+}$ which results from a unitary time development of a particle with $S_{z}=+1 / 2$ interacting with an apparatus arranged to measure $S_{x}$. It is not possible to replace the $U^{+}$and $U^{-}$nodes in Fig. 2(b) with pairs of $X^{+}$and $X^{-}$nodes without violating the standard consistency conditions $[10]$.

Thus consistent quantum counterfactual reasoning requires the specification of a consistent family or framework, as does "ordinary" quantum reasoning [12]. Even in a classical stochastic context, English counterfactual questions are sometimes ambiguous ("What would have happened if the particle had not arrived at $D_{1}$ ?") because the pivot is not specified, and in the quantum context the ambiguity can be even worse, because the English wording ("What would have happened if $S_{x}$ had been measured rather than $S_{z}$ ?") can often be modeled by different frameworks, as well as by different pivots within the same framework 
13. The confusion caused by this ambiguity is illustrated in the following counterfactual version [14, 15] of Hardy's paradox.

Imagine two spin half particles in an appropriate entangled spin state sent in opposite directions to two distant detectors, one on the left and one on the right. Each detector has two switch settings, 1 and 2, which determine which spin component will be measured, and the setting is determined by the flip of a quantum coin, incorporated into the detector, just before the arrival of the corresponding particle. A red light on the detector flashes if the spin component in the measured direction is positive, and a green light if it is negative. Each run yields a result such as $1 \mathrm{G} \cdot 2 \mathrm{R}$, meaning that the left detector had a switch setting of 1 and flashed green, and the right detector with a switch setting of 2 flashed red. The entangled particle state [14] is such that $1 \mathrm{G} \cdot 2 \mathrm{G}, 2 \mathrm{G} \cdot 1 \mathrm{G}$ and $1 \mathrm{R} \cdot 1 \mathrm{R}$ cannot occur (zero probability); all other possibilities occur with finite probability.

To construct a paradox, consider a run in which $2 \mathrm{G} \cdot 2 \mathrm{G}$ is observed and ask what would have happened in this particular experimental run if the quantum coin associated with the right detector had produced a switch setting of 1 rather than 2 . In the absence of long-range influences (in a gedanken experiment, detectors can be arbitrarily far apart!), a last minute change in the switch setting on the right cannot influence the detector on the left, which would, therefore, have flashed green. But since $2 \mathrm{G} \cdot 1 \mathrm{G}$ never occurs, we conclude that if the switch on the right had been 1 , the result in this run would have been $2 \mathrm{G} \cdot 1 \mathrm{R}$. Applying the same argument with the roles of the two detectors interchanged, we conclude that if the switches had been 1 and 2 on left and right, the result would have been $1 \mathrm{R} \cdot 2 \mathrm{G}$. But then, continuing the argument, had both switches been 1, the result would have been $1 \mathrm{R} \cdot 1 \mathrm{R}$, contradicting the fact that $1 \mathrm{R} \cdot 1 \mathrm{R}$ never occurs. Contradictions of this sort easily gives rise to the idea that quantum mechanics involves some mysterious nonlocality: changing the switch setting on the right really does influence the particle or the apparatus on the left in some way.

The basic counterfactual question which must be addressed in thinking about this paradox is the following: given a case in which $2 \mathrm{G} \cdot 2 \mathrm{G}$ is observed, what would have occurred had both of the switch settings been equal to 1? In order to study it using the system of counterfactual reasoning introduced above, one needs to find a pivot at a time which precedes both coin flips, since one is comparing cases in which both of them turned out to be the reverse of what actually occurred. This pivot must involve a quantum state of the two-particle system, since at this earlier time the apparatus states are not playing a significant role. The successive counterfactual steps in the preceding paragraph can then be thought of as devices for finding such a pivot. Viewed in this way, one finds that even the first step of the argument is problematical. In order to infer $2 \mathrm{G} \cdot 1 \mathrm{R}$ from $2 \mathrm{G} \cdot 2 \mathrm{G}$, one must use an earlier state of the two particles which provides a null counterfactual for the left detector: that is, which leads with probability one to the conclusion that its light would (certainly) have flashed green. Simply using the wave function of the original two-particle state as it develops unitarily in time will not support such a sharp conclusion, as there is then a finite probability of $2 \mathrm{R}$ for the left detector [16]; see the previous discussion of Fig. 2(a). A framework in which at an earlier time the left particle is in one of the two states corresponding to the results $2 \mathrm{G}$ and $2 \mathrm{R}$ of a later measurement, analogous to the $z^{+}$and $z^{-}$states of Fig. 2(b), will yield the desired null counterfactual for the left detector. But there is a price to be paid: when the switch on the left detector is changed from 2 to 1 , quantum consistency requires 
the use of an MQS state for the left detector - see the preceding discussion associated with Fig. 2(b) - which will obviously prevent completing the full counterfactual argument of what would have happened had both switches been 1. Note that the MQS problem arises in the course of constructing a correct quantum (counterfactual) description of a single detector, the one on the left, not both detectors. Nonlocality plays no role: what matters is consistent quantum counterfactual reasoning applied to a local measurement.

The framework just discussed is not unique; there are various possibilities which can be used to justify different steps in the counterfactual argument. However, there is no way to combine these into a single framework, as required by the rules of counterfactual reasoning introduced above, in order to complete the entire counterfactual argument in which both switches are changed from 2 to 1 . Were there such a framework it would imply that $1 \mathrm{R} \cdot 1 \mathrm{R}$ can occur with positive probability, at least as large as the probability of $2 \mathrm{G} \cdot 2 \mathrm{G}$. But standard quantum mechanics gives probability zero for $1 \mathrm{R} \cdot 1 \mathrm{R}$, as does any framework or consistent family in which the probability of $1 \mathrm{R} \cdot 1 \mathrm{R}$ makes sense [17]. Hence there cannot be a single framework in which the counterfactual inference leading to a paradox is valid. From this perspective, the Hardy paradox can be thought of as illustrating one of the ways in which classical reasoning can fail when applied in a quantum context.

Consistent quantum counterfactuals can generate positive results as well as block paradoxes. Consider the well-known EPR-Bohm arrangement in which two spin-half particles in a singlet state fly apart, and the $x$ component of the spin of the particle on the right is measured to be $S_{x}=+1 / 2$. Assuming that the particle on the left continues on its trajectory without interacting with anything, one can infer (probability 1 ) that it has $S_{x}=-1 / 2$ both before and after the spin measurement on the right 18. But would it still have had $S_{x}=-1 / 2$ if $S_{z}$ instead of $S_{x}$ had been measured for the particle on the right? The answer is "yes" if one adopts a framework in which $S_{x}$ values for the particle on the left make sense at different times [19]. The argument is straightforward, and only requires combining a previous consistent history analysis [18] of EPR-Bohm (without using counterfactuals) with the proposal in the present article. Again, there is no indication of any mysterious nonlocal influence.

In addition, counterfactual definitions of quantum properties are possible under appropriate circumstances. The analysis given in Sec. 5 of [20] indicates that as long as the behavior of a closed quantum system is described using a consistent family, the corresponding events can, in principle, be checked by idealized measurements. If these are thought of as carried out with the help of appropriate quantum coins, the procedure for counterfactual reasoning given above supports a realistic interpretation of the corresponding quantum events: they would still have occurred even if no measurements had been made [21.

In conclusion, the system of consistent quantum counterfactuals presented here, while of limited scope, is sufficiently powerful to deal with a number of non-trivial issues in quantum foundations, and could well prove to be a useful tool for getting rid of some of the ghosts which have long plagued that discipline.

Conversations and correspondence with R. Clifton, P. Eberhard, B. d'Espagnat, N. D. Mermin, and H. P. Stapp are gratefully acknowledged, together with comments from various anonymous referees, and financial support from the National Science Foundation through grant PHY 96-02084. 


\section{References}

[1] There is an enormous and ever increasing literature devoted to this subject. Various items in [2] are extremely helpful. Critical analyses and references to the literature will be found in [3] and [4]

[2] J. S. Bell, Speakable and Unspeakable in Quantum Mechanics (Cambridge University Press, Cambridge, 1987).

[3] M. Redhead, Incompleteness, Nonlocality, and Realism (Clarendon Press, Oxford, 1987).

[4] B. d'Espagnat, Veiled Reality (Addison-Wesley, Reading, Mass., 1995).

[5] L. Hardy, Phys. Rev. Lett. 68, 2981 (1992).

[6] The use of counterfactuals for discussing the EPR paradox goes back to H. P. Stapp, Phys. Rev. D3 1303 (1971); P. Eberhard, Il Nuovo Cimento B38, 75 (1977); B46, 392 (1978); H. P. Stapp, ibid. B40, 191 (1977), and has proven to be very controversial. See the references in M. Dickson and R. Clifton, Phys. Rev. A 49, 4251 (1994), and H. P. Stapp, ibid. 49, 4257 (1994). Also see [14, 15].

[7] B. d'Espagnat, Phys. Repts. 110, 201 (1984); Reality and the Physicist (Cambridge University Press, Cambridge, 1989).

[8] Counterfactuals in the non-quantum-mechanical context have been extensively explored by philosophers; see, for example, W. L. Harper, R. Stalnaker and G. Pearce, editors, Ifs: Conditionals, Belief, Decision, Chance, and Time (D. Reidel Publishing Co., 1981); D. Lewis, Philosophical Papers (Oxford University Press, 1986).

[9] R. Omnès, The Interpretation of Quantum Mechanics (Princeton University Press, Princeton, 1994).

[10] R. B. Griffiths, Phys. Rev. A 54, 2759 (1996).

[11] This is the quantum counterfactual counterpart to the "single logic", p. 163 of [9], or "single framework", Sec. III of [10], rule for ordinary quantum reasoning.

[12] For comments on how frameworks are to be chosen in the case of ordinary (noncounterfactual) quantum reasoning, see R. B. Griffiths, Phys. Rev. A 571604 (1998).

[13] If the initial state, pivot, and various later events are all specified, the probabilities of interest will be independent of which framework contains these events, for reasons noted in Sec. 5.4 of [9] and Sec. IV of [10]. Note, in particular, the need for an explicit pivot.

[14] N. D. Mermin, Am. J. Phys. 62, 880 (1994). The argument given here differs in some details from that of Mermin, but the basic idea is similar. 
[15] A somewhat different counterfactual formulation of Hardy's paradox by H. P. Stapp, Am. J. Phys. 65, 300 (1997), uses certain assumptions about locality, including Lorentz invariance, in order derive a contradiction, and from this concludes that locality or Lorentz invariance must fail in the quantum world. A proper comparison of Stapp's formulation with the counterfactual analysis in the present article is difficult in the absence of a relativistic generalization of the latter. (I am indebted to H. P. Stapp for lengthy correspondence on this point.)

[16] Quite generally, if one is limited to the consistent families employed in textbook versions of quantum theory, for which measurement outcomes are only related by probabilities to prior states of the measured system, counterfactual reasoning cannot come to very sharp conclusions. Thus within this context, Redhead's criticism, Sec. 4.2 of [3], of earlier counterfactual arguments by Stapp and Eberhard (see [6]) appears to be justified.

[17] See the discussion following (4.5) in [10] about probabilities being independent of refinement.

[18] R. B. Griffiths, Am. J. Phys. 55, 11 (1987).

[19] This provides at least a partial response to a criticism of the consistent history approach by B. d'Espagnat in Waves, Information and Foundations of Physics: a Tribute to Giuliano Toraldo de Francia on his 80th Birthday (Italian Physical Society, Bologna, 1998).

[20] R. B. Griffiths, J. Stat. Phys. 36, 219 (1984).

[21] For some further remarks about quantum reality, and the way in which it differs from its classical counterpart, see [12]. 